\title{
A Systematic Approach in Restructuring Elective Breast \& Endocrine Cancer Surgery during COVID-19 Pandemic in Malaysia
}

\author{
Tan Yee Ling ${ }^{1 *}$, Aminnur HM ${ }^{1}$, Nor Safariny Ahmad ${ }^{1}$, Sadhana Mahamad ${ }^{1}$, Anita \\ Baghawi $^{1}$, Jamil Omar ${ }^{2}$ and Suhaila Md Hanapiah ${ }^{3}$
}

${ }^{1}$ Breast and Endocrine Unit, Department of Surgery, Hospital Putrajaya, Ministry of Health, Malaysia

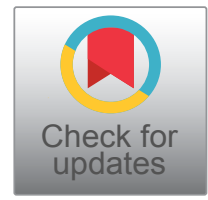

${ }^{2}$ Department of Gynae-Oncology, National Cancer Institute, Ministry of Health, Malaysia

${ }^{3}$ Department of Microbiology \& Pathology, National Cancer Institute, Ministry of Health, Malaysia

*Corresponding author: Tan Yee Ling, General Surgeon, Breast and Endocrine Unit, Department of Surgery, Hospital Putrajaya, Ministry of Health, Malaysia, Tel: +60127877919

\begin{abstract}
The COVID-19 pandemic had resulted in major disruptions in healthcare globally. As a response to the outbreak, global elective surgeries were suspended to preserve resources. To date, Malaysia's effectiveness in tackling the COVID-19 was an instructive example for many East Asia countries. In addition to the public health preparedness, we report our experience in early restructuring of elective breast and endocrine cancer surgery to ensure a continuum in cancer care during the peak of the pandemic.
\end{abstract}

\section{Keywords}

Elective surgery, Breast and endocrine cancer, COVID-19 pandemic, Malaysia

\section{Introduction}

On March 11, 2020, the World Health Organization (WHO) officially declared the novel Coronavirus Disease (COVID-19) pandemic as an international emergency. This disease that began in Hubei Province, China in late December 2019 had since infected more than 5 million individuals globally and approximately 7000 individuals in Malaysia, at the time of drafting this editorial. In response to the pandemic crisis, the federal government of Malaysia had implemented a Movement Control Order (MCO) since 18 March 2020 as a strategy to flatten the curve and to slow the virus spread [1]. The Ministry of Health (MOH) Malaysia had promptly identified 35 designated hospitals to manage suspected/confirmed COVID-19 cases across the states [2]. Government hospitals were categorized into screening hospitals and treating hospitals respectively, leaving few being nonCOVID-19 hospitals. In the effort to minimize the risks of exposure among patients and to preserve resources in anticipation of an overwhelming surge in COVID-19 cases, $\mathrm{MOH}$ issued directives to temporary suspend all elective surgeries starting from 24 March 2020 [3]. This had created a difficult dilemma to both healthcare professionals and patients; especially in those focused on the treatment of cancer. The inherent risks of delaying cancer surgery would inadvertently affect its long term outcome while cancer treatment during this period could lead to an increased risk of infection. Therefore, it is important for the surgeon to balance the risks between potential viral transmission and possible cancer progression [4]. To mitigate such negative impact, a collaborative effort was made to restructure elective breast and endocrine cancer surgery from Hospital Putrajaya (a designated screening COVID-19 hospital) to the nearest non-COVID-19 hospital, the National Cancer Institute. The sole objective was to deliver a safe and high quality care in continuum of oncology surgical services for breast and endocrine cancer patients, amidst an evolving pandemic situation.

\footnotetext{
Citation: Ling TY, Aminnur HM, Ahmad NS, Mahamad S, Baghawi A, et al. (2020) A Systematic Approach in Restructuring Elective Breast \& Endocrine Cancer Surgery during COVID-19 Pandemic in Malaysia. Int J Surg Res Pract 7:117. doi.org/10.23937/2378-3397/1410117

Accepted: October 29, 2020; Published: October 31, 2020

Copyright: (c) 2020 Ling TY, et al. This is an open-access article distributed under the terms of the Creative Commons Attribution License, which permits unrestricted use, distribution, and reproduction in any medium, provided the original author and source are credited.
} 


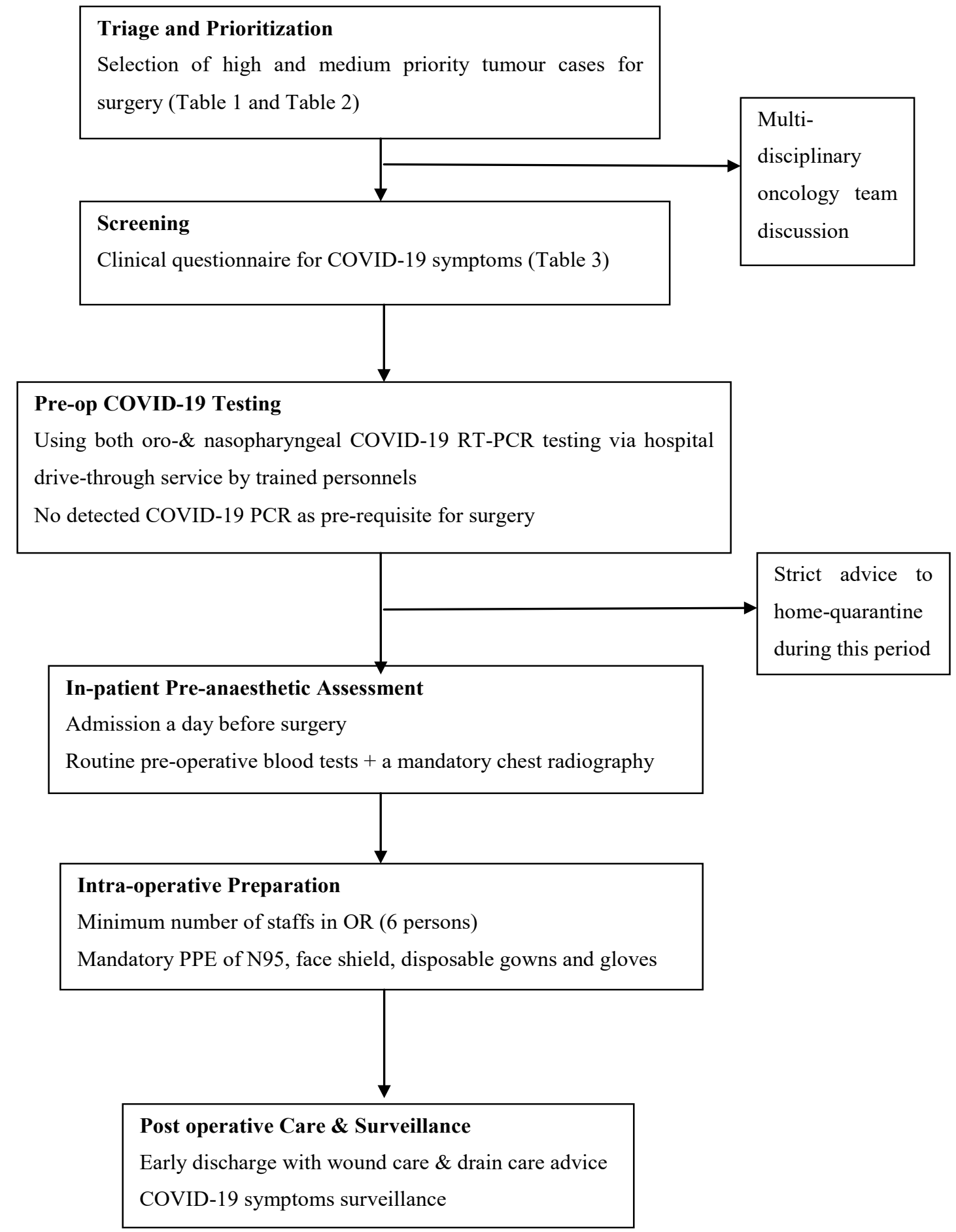

Figure 1: A systematic approach on restructuring breast and endocrine elective cancer surgery from Hospital Putrajaya to National Cancer Institute during COVID-19 pandemic.

\section{Methodology}

A systematic algorithm (Figure 1) was drafted prospectively with adaptation from international recommendations $[5,6]$ by a group of consultant surgeons, anaesthetists, microbiologists/pathologists as well as hospital administrative stakeholders. Six key components of the restructuring are: Triage $\&$ prioritization, clinical health screening, pre-operative COVID-19 testing for all patients, in-patient pre-anaesthetic assessment, intraoperative preparation, post-operative home care with symptoms surveillance. A special task force was formed to coordinate the entire process; ensuring a smooth and safe transition of patients care from one hospital to another (Table 1, Table 2 and Table 3).

\section{Results}

A total number of 85 elective breast and endocrine surgeries that were scheduled from 24 March 2020 onwards were subjected to cancellation or postponement following $\mathrm{MOH}$ directives. With the implementation of this systematic approach to shift elective surgery to a non-COVID-19 hospital, we successfully performed 34 out of 85 cases $(40.0 \%)$ during our country's peak pandemic crisis from 30 March 2020 to 22 May 2020 (8 
Table 1: Triage \& Prioritization of breast tumour cases for surgery tailored to local case-mix (adapted from ACS [11], ESMO [12]) (ACS-American College of Surgeons, ESMO-European Society of Medical Oncology).

\begin{tabular}{|l|l|}
\hline Breast tumour & Completed post-neoadjuvant chemotherapy \\
\hline High priority & Complications of prior breast cancer surgery with bleeding or infection requiring re-intervention \\
\hline & Complications from reconstructive surgery \\
\hline & $\begin{array}{l}\text { Breast cancer diagnosed in pregnancy } \\
\text { Unusual cases for special considerations: } \\
\text { bleeding/ruptured tumours }\end{array}$ \\
\hline Triple negative /HER2+ \\
\hline High grade DCIS \\
\hline Discordant biopsy of likely to be malignant \\
\hline Medium priority & Malignant or local recurrence \\
\hline Axillary surgery staging \\
\hline Low priority & Low grade DCIS \\
\hline Discordant biopsy likely benign \\
\hline Breast reconstruction (autologous \\
\hline Excision of benign breast lesion \\
\hline Prophylactic surgery for high risk asymptomatic patients \\
\hline
\end{tabular}

*Triple negative in patients who were either not indicated or not fit for systemic chemotherapy

Table 2: Triage \& Prioritisation of endocrine tumour cases tailored to local case-mix (adapted from ACS).

\section{Endocrine tumour}

\begin{tabular}{|c|c|}
\hline \multirow[t]{4}{*}{ Thyroid } & Goiter causing airway compromise \\
\hline & Life-threatening or severely symptomatic Graves' that cannot be controlled medically \\
\hline & $\begin{array}{l}\text { Thyroid cancers that are imminently threatening the life or the health of the patient such as those with short } \\
\text { double timing or aggressive recurrences }\end{array}$ \\
\hline & $\begin{array}{l}\text { Open biopsy to confirm a suspected diagnosis of anaplastic thyroid cancer or thyroid lymphoma in order to } \\
\text { direct appropriate treatment }\end{array}$ \\
\hline Parathyroid & Hyperparathyroidism with life threatening hypercalcemia not medically controlled \\
\hline \multirow[t]{2}{*}{ Adrenal } & Suspected adrenal cortical carcinoma \\
\hline & Pheochromocytoma or paraganglioma that cannot be controlled medically \\
\hline General & $\begin{array}{l}\text { Endocrine disorders in pregnant patient that are dangerous to the health of the mother or fetus that cannot be } \\
\text { controlled medically }\end{array}$ \\
\hline
\end{tabular}

Table 3: Screening questionnaires adapted from Health Declaration Form, Ministry of Health Malaysia.

\section{Clinical screening questionnaires}

\begin{tabular}{|c|c|c|c|c|}
\hline & \multicolumn{4}{|c|}{ Do you have any of the following (new or worsening symptoms) of acute respiratory infection? } \\
\hline & DFever & DShortness of breath & DCough & DSore throat \\
\hline & AND & & & \\
\hline \multirow[t]{7}{*}{ PUI of COVID-19 } & \multicolumn{4}{|c|}{ Have you travelled/resided in a foreign country within 14 days before the onset of illness } \\
\hline & DYes & \multicolumn{3}{|l|}{ DNo } \\
\hline & \multicolumn{4}{|c|}{ Close contact in 14 days before illness onset with confirmed case of COVID-19 } \\
\hline & DYes & \multicolumn{3}{|l|}{ DNo } \\
\hline & \multicolumn{4}{|l|}{ OR } \\
\hline & \multicolumn{4}{|c|}{ Attended an event associated with known COVID-19 outbreak } \\
\hline & DYes & \multicolumn{3}{|l|}{ DYes } \\
\hline
\end{tabular}

weeks) (Table 4). Only cases of high to medium priority were selected for surgery. All patients had passed their preliminary clinical health screening questionnaires for
COVID-19 symptoms via telecommunications, prior to the diagnostic COVID-19 swabs tests. Similarly, all patients were confirmed negative for COVID-19 PCR prior 
Table 4: Type of surgery and total number of cases successfully performed electively.

\begin{tabular}{|l|l|l|}
\hline Organ & Type of surgery & No. of cases \\
\hline \multirow{3}{*}{ Breast } & Mastectomy +/- Axillary clearance & 18 \\
\cline { 2 - 3 } & Wide local excision \& Axillary clearance (Breast conserving surgery) & 4 \\
\cline { 2 - 3 } & Mastectomy + Sentinel lymph node biopsy & 4 \\
\cline { 2 - 3 } & Removal of infected implant failing to respond to prolonged antibiotics & 1 \\
\cline { 2 - 3 } & Wide local excision for suspected malignancy & 1 \\
\hline \multirow{2}{*}{ Thyroid } & Excision of chest wall recurrence/Axillary lymph node recurrence & 2 \\
\hline & Total thyroidectomy +/- Modified radical neck dissection + Tracheostomy & 1 \\
\hline Total & Total thyroidectomy for multinodular goitre with airway obstruction in pregnancy & 2 \\
\hline
\end{tabular}
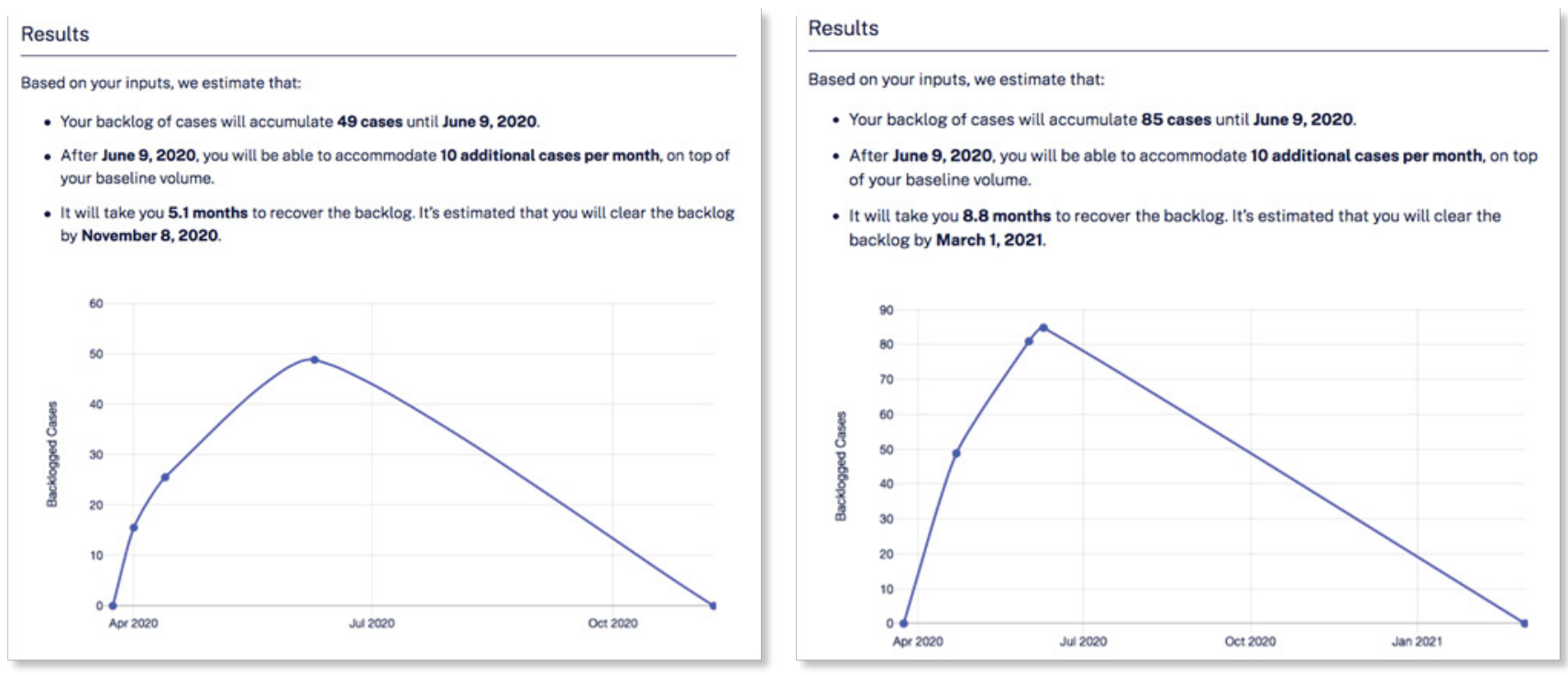

Figure 2: $(a, b)$ Estimating total time taken to recover from backlog of cases using iQueue elective surgery backlog recovery calculator between 49 cases vs. 85 cases (5.1 months vs. 8.8 months).

to surgeries. Neither patients nor health care workers developed COVID-19 symptoms/disease throughout this period of hospital admission and surgery.

\section{Discussion}

Hospital Putrajaya is one of the three highest volume tertiary breast and endocrine centre in Malaysia. Statistically, our hospital manages approximately 600 elective breast and endocrine surgeries annually; averaging 12 cases per week. The impact of elective surgery cancellation would undoubtedly add to a growing backlog that needs to be addressed once the pandemic is over. The CovidSurg Collaborative Researchers from Birmingham recently estimated $\mathbf{2 8 . 4}$ million elective surgeries will be cancelled or postponed worldwide during the peak 12 weeks disruption due to COVID-19. This data was then statistically modelled to estimate totals for cancelled surgery across 190 countries; generating an overall 12 -week cancellation rate to be $72.3 \%$ with 2.3 million cases $(37.7 \%)$ being cancer surgery. If countries increase their normal surgical volume by $20 \%$ post-pandemic, it would take a median of 45 weeks ( 11 months) to clear the backlog of operations resulting from COVID-19 disruption [7]. Using a similar iQueue Elective Surgery Backlog Recovery Calculator [8], it estimated 8.8 months were required to clear the backlog if all our cases ( $n=$ $85)$ were postponed. Following this early restructuring, we were able to reduce our backlog and time- predicted to recover to 5.1 months (Figure $2 \mathrm{a}$ and Figure $2 \mathrm{~b}$ ). Thus, cancellation rate was reduced to $60 \%$. However, resuming elective cancer surgery was not without its risks. As cancer patients were considered as high risk individuals, it is crucial to provide them adequate information regarding potential serious adverse events that could occur during an elective surgery. Evidence was scarce and largely retrospective then; Shaoqing, et al. [9] studied 34 patients that have surgery during the incubation of COVID-19 infection which carried a mortality rate of $20.5 \%$. But his report was limited by the small number of representation of only five cancer cases and all patients had prior direct exposure to Wuhan City. Liang, et al. [10] with the largest prospective cohort COVID-19 patients ( $n=1571)$, reported that cancer is associated with an increased risk of death and/or intensive care unit admission in COVID-19 disease (odds-ratio of 5.4, 95\% Cl 1.8-16.2). But due to the small number of 18 cancer 
cases in his study, it did not establish a definitive correlation of increase in incidence of cancer with COVID-19 infection. By far, we had no COVID-19 infection in our 34 cancer patients that underwent surgery.

Two samples of nasopharygeal and oropharyngeal swabs were performed in each patient prior to surgery. The Abbott Realtime SARS-COV-2 assays that were used have $100 \%$ sensitivity. Our patients were subjected to a screening test five days prior to surgery following which they were strictly self-quarantined in line with the MCO. A negative result was a pre-requisite to undergo elective surgery under general anaesthesia. A positive result would bar the patient from surgery and treatment as per COVID-19 patient. Surgery would then be carried out following clearance of two negative swabs posttreatment. All surgeries were performed under general anaesthesia in full Personal Protective Equipment (PPE) with biohazard measures implemented. Intubations were performed with 'intubation box' following which patients and machines were covered with plastic sheets to reduce droplet spread. Restricting number of staffs to only six personnel (i.e. one surgeon, one assistant surgeon, one anaesthetist, one anaesthetist medical officer, one scrub nurse and one circulating nurse) to minimise exposure to staffs. Post-operatively, patients were discharged as early as day one if feasible. Home surveillance by daily telecommunication as well as to follow up on any development of COVID-19 symptoms [11,12].

\section{Conclusion}

The COVID-19 disease outbreak had resulted in many uncertainties in all sectors of health care management. As the elective surgeries are slowly being reintroduced globally, we were fortunate to have had first-hand experience in triaging, prioritizing and treating cancer patients that required elective cancer surgery care during the peak disruption. This systematic approach served as an exemplary to safely resume elective surgery within the limits of national protocols as the pandemic gradually abates. The need to strategize, restructure and sustain cancer surgery services should always be balanced against patient's safety.

\section{References}

1. Movement control order.

2. Guidelines COVID-19 management in Malaysia

3. Guidelines on management of coronavirus disease 2019 (COVID-19) in surgery.

4. Bartlett DL, Howe JR, Chang G, Crago A, Hogg M, et al. (2020) Management of cancer surgery cases during the COVID-19 pandemic: Considerations. Ann Surg Oncol 27: 1717-1720.

5. Hanna TP, Evans GA, Booth CM (2020) Cancer, COVID-19 and the precautionary principle: Prioritizing treatment during a global pandemic. Nat Rev Clin Oncol 17: 268-270.

6. Zheng Liu, Yawei Zhang, Xishan Wang, Daming Zhang, Dechang Diao, et al. (2020) Recommendations for surgery during the novel coronavirus (COVID-19) epidemic. Indian J Surg 82: 124-128.

7. Dmitri Nepogodiev, Omar M Omar, James C Glasbey, Elizabeth $\mathrm{Li}$, Joana FFS, et al. (2020) Elective surgery cancellations due to the COVID-19 pandemic: Global predictive modelling to inform surgical recovery plans. Br J Surg.

8. Elective surgery backlog recovery calculator, iQueue.

9. Shaoqing Lei , Fang Jiang, Wating Su, Chang Chen, Jingli Chen, et al. (2020) Clinical characteristics and outcomes of patients undergoing surgeries during the incubation period of COVID-19 infection. E Clinical Medicine 21.

10. Wenhua Liang, Weijie Guan, Ruchong Chen, Wei Wang, Jianfu Li, et al. (2020) Cancer patients in SARS-CoV-2 infection: A nationwide analysis in China. Lancet Oncol 21: 335-337.

11. COVID-19 Guidelines for triage of breast cancer patients.

12. ESMO Management and treatment adapted recommendations in the COVID-19 era: Breast cancer. 